Check for updates

Cite this: RSC Adv., 2017, 7, 22640

Received 25th January 2017

Accepted 15th April 2017

DOI: $10.1039 / c 7 r a 01108 c$

rsc.li/rsc-advances

\section{Evaluation of the protective effects of 13 traditional Chinese medicine compounds on ionizing radiation injury: bupleurum, shenmai, and breviscapine as candidate radioprotectors}

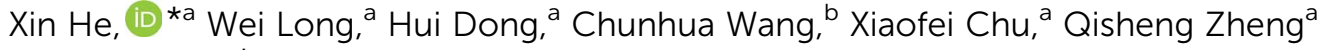
and Saijun Fan*a

Background: Drugs are being sought that are effective as radioprotection for use in both planned and unplanned radiation exposure. Traditional Chinese medicines have been used successfully for the treatment of free radical-mediated diseases and therefore we expected that exploring such agents could yield an effective radioprotector. We therefore evaluated the radioprotective effects of 13 traditional Chinese medicine compounds (TCMCs) against ionizing radiation damage. Materials and methods: TCMCs were tested in vitro on human kidney 293 T cells treated with 0, 2, 4, 6, 8 or 10 Gy irradiation for their ability to promote cell survival. Mice were subjected to a sub-lethal (4 Gy), lethal (7 Gy) or supralethal (13 Gy) dose of total-body irradiation (TBI) and TCMCs were tested for their ability to influence survival and hemopoietic function. Results: Of the 13 TCMCs tested, five including bupleurum, shenmai, and breviscapine, promoted survival of 293T cells. bupleurum, shenmai, and breviscapine also prolonged survival times of mice treated with supra-lethal and lethal TBI doses. As expected of an effective radioprotector, they also ameliorated bone marrow suppression, reducing the decrease in bone marrow cells after sub-lethal TBI, and they attenuated the TBI-induced reduction in granulocyte-macrophage colony-forming units. Conclusion: This investigation of TCMCs in vitro and in vivo identified bupleurum, shenmai, and breviscapine as having characteristics consistent with radioprotective agents. Further investigation to identify the bioactive components responsible for the radioprotective effect of these compounds is now required.
\end{abstract}

\section{Introduction}

Over the past decades, scientists worldwide have been searching for drugs that are effective in radioprotection and in radiorecovery for potential application during both planned (e.g., radiotherapy) and unplanned (e.g., nuclear accident, warfare, occupational) radiation exposure. ${ }^{1}$ An ideal radioprotector needs to meet prerequisite characteristics, such as having no cumulative or irreversible toxicity, an effective and definitive effect, stability (a 25 year shelf-life), and an easy administration route. ${ }^{2}$ So far, no single agent meets all the requirements. Amifostine (trade name Ethyol) is the only radioprotector that has been approved for use clinically, for the radiotherapy of head and neck cancer, despite its drawbacks of short term, and severe side effects (e.g., nausea,

aTianjin Key Laboratory of Radiation Medicine and Molecular Nuclear Medicine, Institute of Radiation Medicine, Peking Union Medical College \& Chinese Academy of Medical Sciences, 238 Baidi Road, Nankai District, Tianjin 300192, China.E-mail: hexin@irm-cams.ac.cn; fansaijun@irm-cams.ac.cn; Fax: +86-2285683033; Tel: +86-22-85682357; +86-22-85685301

${ }^{b}$ Tianjin Key Laboratory of Modern Chinese Medicine, Tianjin University of Traditional Chinese Medicine, 312 Anshanxi Road, Nankai District, Tianjin 300193, China vomiting, diarrhea, hypotension, hypo-calcemia, nephro- and neuro-toxicity). Thus, there is an urgent need in radiation medicine for radioprotective agents with less toxicity and greater efficacy, and the quest to find them is ongoing.

A number of traditional Chinese medicines (TCMs) have been used successfully for the treatment of free radicalmediated diseases such as rheumatoid arthritis, atherosclerosis, inflammatory diseases, and ischemia/reperfusion damage., It is therefore reasonable to expect that there is a potential single or combination of different radiomodifiers that would be successful against ionizing radiation injury. The present study attempts to evaluate the potential radioprotective efficacy of 13 traditional Chinese medicine compounds (TCMCs) via cell culture, and animal models of total-body irradiation (TBI) in the sub-to supra-lethal range.

\section{Materials and methods}

\subsection{Experimental materials}

Detailed information on the 13 TCMCs are listed in Table 1. All are herbal products exhibiting anti-inflammatory properties or 


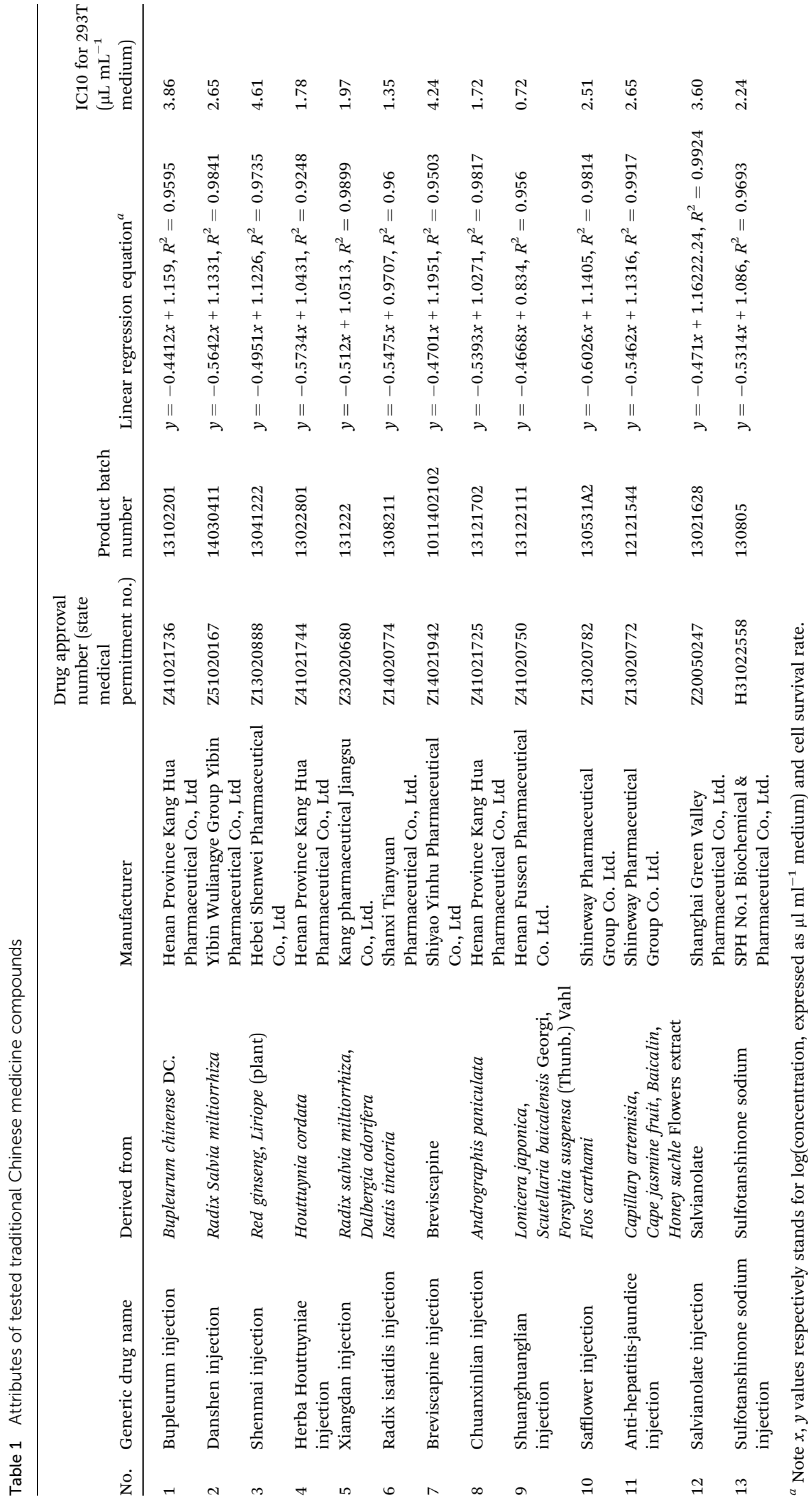


anti-microbial effects. Drug dosages for use in the experiments were selected according to those already used in clinical applications; the human to mouse equivalent dose rate was calculated according to body surface area. ${ }^{5}$ Medicines with single active ingredient compound use doses in $\mathrm{mg}$ per $\mathrm{kg}$ per day and others with complex active ingredients in $\mathrm{mL}$ per $\mathrm{kg}$ per day. Different doses of TCMCs dissolved in cell culture medium (for use in vitro) or saline (for use in vivo) were freshly prepared prior to each experiment. Unless otherwise specified, all of the chemicals used in this study were obtained from Sigma Aldrich (St Louis, MO, USA). Prior to use, stock solutions were stored at $4{ }^{\circ} \mathrm{C}$ in the dark. Radiation exposure was performed using a Gammacell-40 ${ }^{137} \mathrm{Cs}$ irradiator (Atomic Energy of Canada Ltd.,

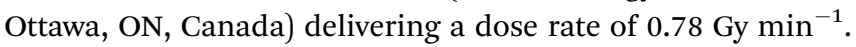

\subsection{Cell culture and MTT assay}

The human embryonic kidney cell-derived cell line 293T was obtained from the American Type Culture Collection (Rockville, MD, USA). Cells were cultured in basic DMEM supplemented with $10 \%$ fetal bovine serum and 100 units per $\mathrm{mL}$ penicillin and streptomycin antibiotic mixture at $37{ }^{\circ} \mathrm{C}$ in a humidified $5 \% \mathrm{CO}_{2}$ atmosphere. Cell growth inhibition was determined using the colorimetric 3-(4,5-dimethyl-2-thiazolyl)-2,5-diphenyl2- $H$-tetrazolium bromide (MTT) assay, which relies on the change from yellow MTT to purple formazan crystals that occurs in the presence of living cells. The assay was conducted in a 96well plate on $8 \times 10^{3}$ cells per $200 \mu \mathrm{L}$ per well. Cells were incubated for $24 \mathrm{~h}$, after which the medium was removed and replaced with fresh medium containing the test compound, TCMCs were serially diluted with cell culture medium, followed by an incubation period of 24 or $48 \mathrm{~h}$. Cells were then incubated with MTT solution $\left(0.5 \mathrm{mg} \mathrm{mL}^{-1}\right)$ for $4 \mathrm{~h}$, carefully remove the supernatant with a pipette leaving behind the precipitate, and then $150 \mu \mathrm{L}$ of dimethyl sulfoxide was added to each well to dissolve formazan precipitate. The absorbance $(A)$ of each well (six parallel wells for each sample) was then measured at $570 \mathrm{~nm}$ using a Synergy HT Multi-Mode Microplate Reader (BioTek, Winooski, VT, USA). The percentage of cells surviving was calculated using the following formula: cell survival rate $(\%)=\left[\left(A_{\text {test }}-A_{\text {blank }} / A_{\text {negative control }}-A_{\text {blank }}\right)\right] \times 100$. These experiments were conducted in triplicate. A graph was constructed by linear regression analysis of the cell survival rate against the $\log$ concentration (expressed as $\mu \mathrm{L} \mathrm{mL}^{-1}$ medium) of the tested compound, which was used to determine the concentration of compound that inhibits $10 \%$ of cells (IC10; data not shown). Drug concentration at an inhibition rate of $10 \%$, which was considered not to affect cell proliferation and growth, we selected the half IC10 concentration considering its toxicity and negative effects on cells were negligible. TCMC was subsequently used as the single dose for further experiments on $293 \mathrm{~T}$ cells detailed below.

\subsection{Colony formation assay}

Colony formation assays were conducted with half IC10 concentrations of each TCMC to test the protective effect of each compound. Cells were seeded into 12-well plates at a density of 1000 cells/well $/ 2 \mathrm{~mL}$ cultured for $24 \mathrm{~h}$ to allow them to stabilize, then TCMCs were added separately followed by $12 \mathrm{~h}$ culture. Cells were then treated with a range of ionizing irradiation (IR) doses $(0,2,4,6,8$ or $10 \mathrm{~Gy})$. After incubating these cells at $37^{\circ} \mathrm{C}$ for 14 days, they were washed twice with phosphate buffered saline (PBS) and stained with $0.005 \%(\mathrm{w} / \mathrm{v})$ crystal violet in $25 \%$ $(\mathrm{v} / \mathrm{v})$ methanol resulted in best staining/background. Using an inverted light microscope to examine the plate wells, the number of colonies containing $\geq 50$ cells were counted, and the number used to calculate: the plating efficiency $(\mathrm{PE})=$ (colonies counted/cells seeded) $\times 100 \%$ and the survival fraction $(\mathrm{SF})=($ colonies counted $) /(($ cells seeded $) \times(\mathrm{PE} / 100))$. GraphPad Prism 5 (GraphPad Software, LaJolla, CA, USA) was used to fit the cell survival curve to a standard linear-quadratic (LQ) model. ${ }^{6}$ The linear-quadratic (LQ) model of radiation effect was used to provides a satisfactory and practically useful explanation of fractionation and dose - rate effects, which can be applied to both tumor and normal tissues. Thereafter, we obtained the values of the $\mathrm{LD}_{50}$ (median lethal dose) of each compound according to this model.

\subsection{Animal grouping, modeling, and administration}

Male C57/BL mice were obtained from the Beijing Vital River Laboratory Animal Technology Co., Ltd. and housed in the certified animal facility at the Institute of Radiation Medicine of the Chinese Academy of Medical Sciences (CAMS) in a specificpathogen-free grade animal facility under standard conditions (temperature of $22 \pm 2{ }^{\circ} \mathrm{C}, 12 / 12 \mathrm{~h}$ light/dark cycle, and humidity of $50 \pm 10 \%$ ) with free access to water. Mice (10-12 weeks old; weighing 22-25 g) were divided randomly into three groups ( $n=10$ in each group): (a) sham-irradiated controls (SI control), receive no saline or TCMC, were placed in identical

Table 2 Nonlinear regression parameters according to linear-quadratic model ${ }^{a}$

\begin{tabular}{|c|c|c|c|c|c|c|c|c|c|c|c|c|c|c|}
\hline Group & IR & $\mathrm{IR}+1$ & $\mathrm{IR}+2$ & $\mathrm{IR}+3$ & $\mathrm{IR}+4$ & $\mathrm{IR}+5$ & $\mathrm{IR}+6$ & $\mathrm{IR}+7$ & $\mathrm{IR}+8$ & $\mathrm{IR}+9$ & $\mathrm{IR}+10$ & $\mathrm{IR}+11$ & $\mathrm{IR}+12$ & $\mathrm{IR}+13$ \\
\hline Best-fit & 0.1041 & 0.0555 & 0.2270 & 0.0598 & 0.0506 & 0.2621 & 0.4416 & 0.0750 & 0.4015 & 0.3142 & 0.3051 & 0.3736 & 0.2519 & 0.0543 \\
\hline values & 0.0163 & 0.0132 & 0.0181 & 0.0132 & 0.0143 & 0.0248 & 0.0095 & 0.0127 & 0.0004 & 0.0183 & 0.0159 & 0.0056 & 0.0183 & 0.0202 \\
\hline$R^{2}$ & 0.9862 & 0.9951 & 0.9944 & 0.948 & 0.9825 & 0.9984 & 0.997 & 0.9754 & 0.9932 & 0.9967 & 0.994 & 0.9994 & 0.9961 & 0.9841 \\
\hline$\alpha / \beta$ & 6.40 & $4.22^{b}$ & 12.52 & $4.53^{b}$ & $3.55^{b}$ & 10.58 & 46.49 & $5.90^{b}$ & 1120.88 & 17.22 & 19.15 & 66.98 & 13.79 & $2.69^{b}$ \\
\hline $\mathrm{LD}_{50}(\mathrm{~Gy})$ & 4.07 & $5.45^{b}$ & 2.54 & $5.33^{b}$ & $5.42^{b}$ & 2.19 & 1.52 & $5.00^{b}$ & 1.72 & 1.98 & 2.05 & 1.81 & 2.35 & $4.67^{b}$ \\
\hline
\end{tabular}

${ }^{a}$ Note: $\mathrm{SF}=\exp \left(-\alpha x-\beta x^{\wedge} 2\right)$ according to linear-quadratic model, SF means survival fraction; $x$ means radiation dose; $\alpha / \beta$ has positive correlation with radiosensitivity. ${ }^{b}$ Lower radiosensitivity compared with IR group. 
Table 3 Drug doses applied in each TBI experiments

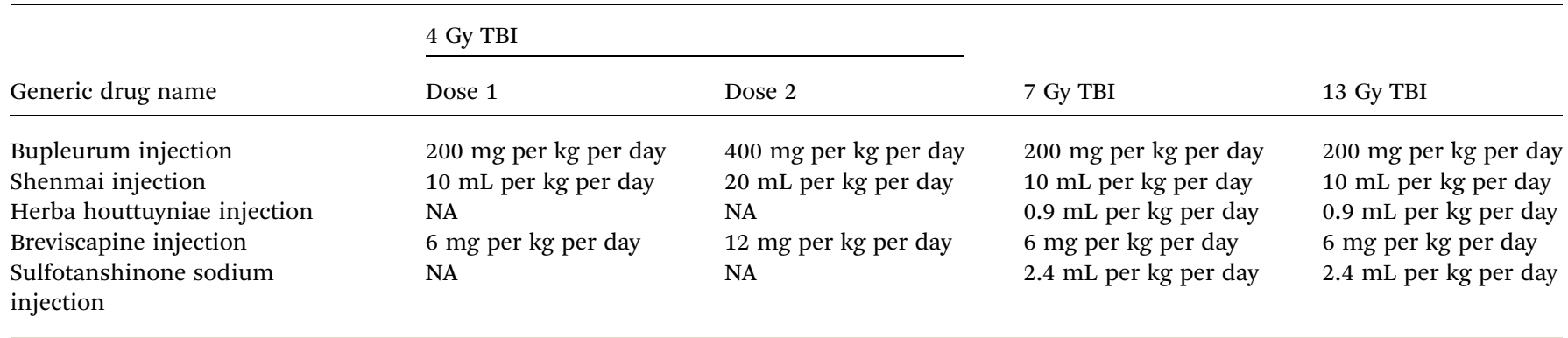

containers as the TBI groups for the same period without irradiation (b) TBI + saline, and (c) TBI + TCMC. The mice in the TBI + TCMC group were injected intraperitoneally with different doses (see Table 3) of TCMC 30 min before and immediately after IR, and were then maintained on the same dose once per day for seven days after IR. The TBI + saline group received the same volume of physiological saline instead of TCMC at the same frequency and duration as the TBI + TCMC group. In the survival assays, mice exposed to a supra-lethal dose of 13 Gy or a lethal dose of 7 Gy TBI were returned to the animal facility for daily observation and treatment as indicated. Treated mice were observed twice or three times daily for a period of 30 days and their body weight and survival condition recorded. The mice that received a sub-lethal dose of 4 Gy TBI were sacrificed two days after the seven-day treatment period and specimens were collected. All experiments were performed in compliance with Guidelines of Animal Experiments from Medical Ethics Committee, National Health Department of China, and were approved by the Animal Use Ethics Committee of CAMS.

\subsection{Specimen collection}

Nine days after sub-lethal TBI, peripheral blood $(100 \mu \mathrm{L})$ was obtained from the orbital sinus using a micro-pipette coated with $\mathrm{K}_{3}$ EDTA anticoagulant. A Celltac Es hemocytometer (Nihon Kohden, Japan) was used to count white blood cells (WBCs) red blood cells (RBCs) and platelets (PLT), and measure hemoglobin (HGB) and RBC-specific volume (HCT), in each peripheral blood sample. Bone marrow (BM) cells were flushed from the femurs of the sacrificed mice with PBS, and bone marrow mononucleated cells (BMMNCs) were counted using the Celltac Es hemocytometer and expressed as number of cells per femur.

\subsection{Hematopoietic colony-forming unit assay}

Hematopoietic colony-forming unit assays were conducted by culturing BMMNCs in MethoCult GF M3534 methylcellulose medium (StemCell Technologies, Vancouver, BC, Canada) for 5-7 days; colonies of granulocyte-macrophage cells (CFU-GM) with $>30$ cells were counted.

\subsection{Statistical analysis}

Data are presented as the mean \pm standard error of the mean (SEM). Survival for each group was estimated using KaplanMeier survival curves and differences in the survival curves among different groups were evaluated using the log-rank test.
Differences in blood cell counts between groups were analyzed using one-way ANOVA and the Mann-Whitney $U$ test. A $p$-value of $<0.05$ was considered statistically significant. All tests were two-tailed. All analyses were performed using GraphPad Prism Software and IBM SPSS Statistics version 22.0.

\section{Results}

\subsection{TCMC IC10 values for $293 \mathrm{~T}$ cells}

The toxicity of the 13 TCMCs was determined in 293T cells by analyzing the IC10 values, the experimental standard of noobserved-effect concentration. Linear regression analysis was performed to estimate the relationship between the cell survival rate ( $y$-value), and the log concentration of the tested compound ( $x$-value); the goodness-of-fit values $\left(R^{2}\right)$ ranged from $0.92-0.99$, and are shown with the linear regression equations for each compound in Table 1 . The resulting equations were used to determine the IC10 values, which ranged from $0.72-4.61 \mu \mathrm{L}$ $\mathrm{mL}^{-1}$ medium, with the highest and lowest toxicity being caused by Shuanghuanglian (no. 9) and shenmai (no. 3), respectively (Table 1 ).

\subsection{T cell colony formation}

To investigate the influence of TCMC treatment on cell radiosensitivity, 293T cells were treated with TCMC at the half IC10 concentration followed by $0,2,4,6,8$ or 10 Gy IR and then cultured for 14 days. The SF data were fitted to a LQ model ${ }^{6}$ using the formula $\mathrm{SF}=\exp \left(-\alpha x-\beta x^{\wedge} 2\right)$, where the $x$-value is the radiation dose. The parameters $\alpha$ and $\beta$ are the best-fit values and were calculated by non-linear regression analysis; the $\alpha / \beta$ ratio positively correlates with radiosensitivity. A significantly lower $\alpha / \beta$ ratio than that resulting from IR alone was evident for cells treated with bupleurum (no.1), shenmai (no. 3), herba houttuyniae (no. 4), breviscapine (no. 7), and sulfotanshinone sodium (no. 13; Table 2). The non-linear regression curve (according to LQ model) of the SF at each IR dose in the presence of each TCMC, and the survival curve for cells treated with the five potentially radioprotective TCMC at each dose of IR are shown in Fig. 1a and b, respectively.

\subsection{Effect of TCMCs on survival of mice after lethal and supra-lethal TBI}

To determine whether TCMCs had any radioprotective effect against supra-lethal and lethal doses of TBI in vivo, mice in the 

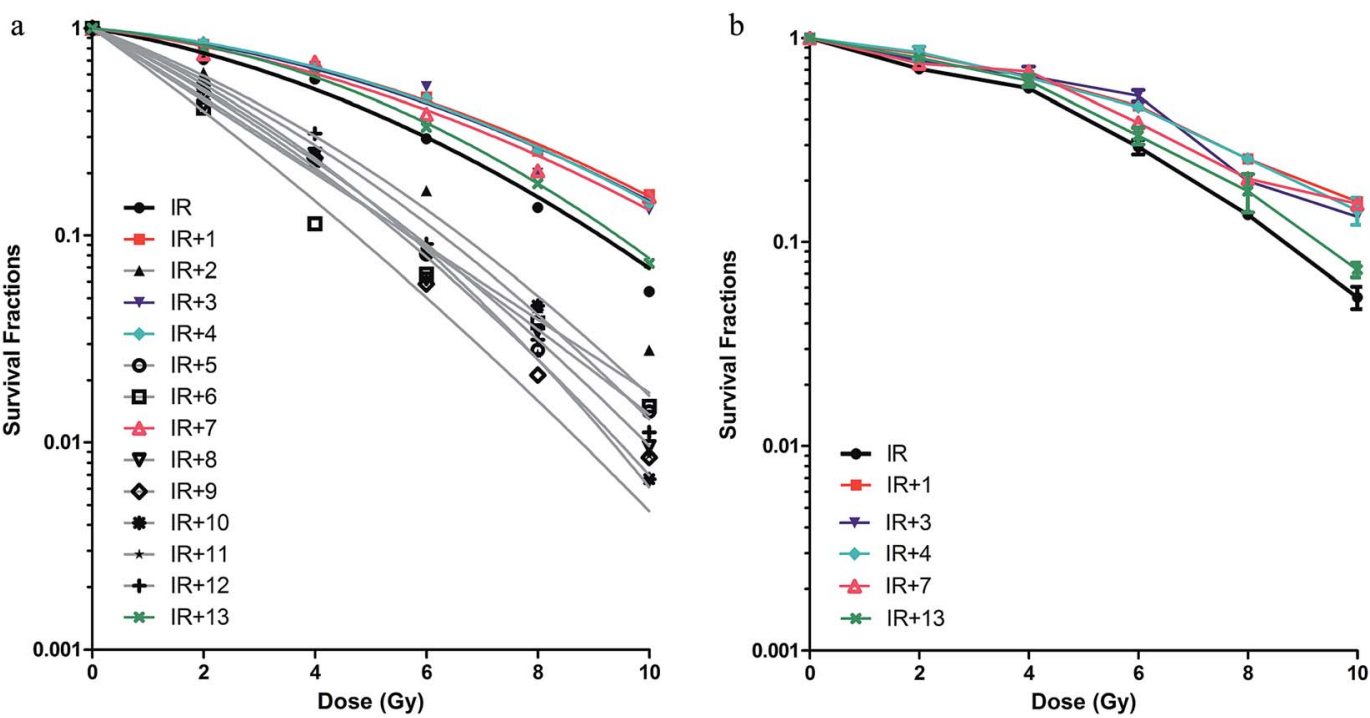

Fig. 11 (Bupleurum injection), 3 (shenmai Injection), 4 (herba Houttuyniae injection), 7 (breviscapine injection), or 13 (sulfotanshinone sodium injection) treatment promotes colony formation ability of $293 \mathrm{~T}$ cells after ionzing radiation. (a): nonlinear regression curve of $293 \mathrm{~T}$ cells survival fractions treated with 0, 2, 4, 6, 8 or 10 Gy of gamma-ray according to Linear-quadratic model; (b): 1 (bupleurum injection), 3 (shenmai injection), 4 (herba houttuyniae injection), 7 (breviscapine injection), 13 (sulfotanshinone sodium injection) promotes survival fractions curve of radiated $293 \mathrm{~T}$ cells.

TCMC test groups were given TCMC (or TBI + saline) before, immediately after a supra-lethal dose of $13 \mathrm{~Gy} \mathrm{TBI}$, and for seven days afterwards, and the survival duration of the mice was monitored. Only data from TCMCs showing any positive effect are presented. Statistical differences between survival times of the TCMC-treated groups and the TBI + saline group were calculated using the log-rank test. The median survival times was 6 in the groups of mice treated with TBI + saline (control), while $8,8,7,7.5$ and 6 days in TBI + bupleurum $(p=0.0007)$, TBI + shenmai $(p=0.034)$, TBI + herba houttuyniae $(p=0.062)$, TBI + breviscapine $(p=0.032)$ and TBI + sulfotanshinone sodium ( $p=0.543$ ) groups, respectively. Next, we performed the same protocol but with a lethal dose of 7 Gy TBI and monitored the mice for up to 30 days after the end of the seven-day treatment period. The median survival times were 16.5, 24.5, 24.5, 24, 24.5 and 16.5 days with 30 day survival rates of $0,50 \%, 50 \%, 50 \%$, a

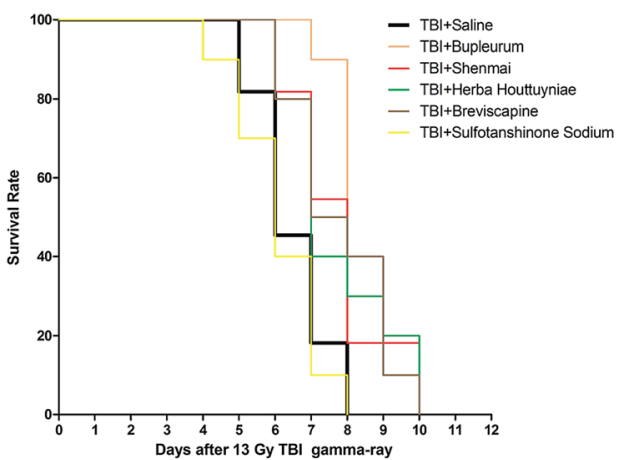

c

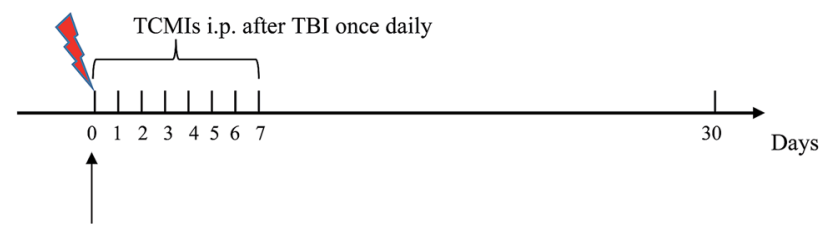

b

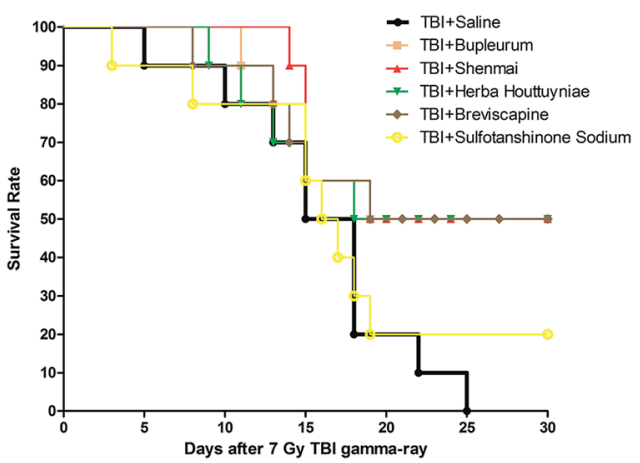

TCMIs i.p. 30 mins before TBI

Fig. 2 Survival curves after supralethal dose and lethal dose TBI. 1 (bupleurum injection), 3 (shenmai injection), 7 (breviscapine injection) prolonged survival time of mice exposured to supralethal ( $13.0 \mathrm{~Gy}$ ) dose TBI ((a), $p=0.0007,0.034,0.062)$, increased the 30 day survival rate of mice exposured to lethal (7.0 Gy) dose TBI ((b), $p=0.036,0.024,0.048)$; (c) TBI and drug treatment. 
$50 \%$ and $20 \%$ in the TBI + saline (control), TBI + bupleurum $(p=$ $0.036)$, TBI + shenmai $(p=0.024)$, TBI + herba houttuyniae $(p=$ $0.064)$, TBI + breviscapine $(p=0.048)$, TBI + sulfotanshinone sodium ( $p=0.55$ ) groups, respectively (Fig. 2 and Tables 4 and 5).

\subsection{Effect of TCMCs on sub-lethal TBI-induced BM suppression in mice}

Exposure to TBI usually induces peripheral blood count inhibition and BM damage. ${ }^{7,8} \mathrm{An}$ effective radioprotector will rescue these parameters. Using the same treatment protocol as detailed for the supra-lethal TBI dose, we therefore evaluated the effects of TCMCs on hematopoietic parameters after exposure to a sub-lethal dose of $4 \mathrm{~Gy} \mathrm{TBI}$. Based on the results of the lethal and supra-lethal TBI experiments, we selected the top 3 positive drugs of the TCMCs with two doses of each (doses applied see Table 3) to evaluate their affection on BM suppression in mice. As shown in Table 5, levels of WBCs, RBCs, HGB, HCT and PLTs in the TBI + saline group were significantly lower than in the SI control group $(p<0.001)$. With TCMC treatment, WBCs, RBCs, HGB and HCT levels were increased but still remained lower than those of the SI controls. Compared to that of the TBI + saline group, the number of BMMNCs was significantly increased in the TBI + bupleurum (200 $\left.\mathrm{mg} \mathrm{kg}^{-1} ; p=0.0012\right)$, TBI + shenmai $\left(10 \mathrm{~mL} \mathrm{~kg}^{-1}\right.$ and 20 $\mathrm{mL} \mathrm{kg}{ }^{-1} ; p=0.049, p=0.0038$, respectively), and TBI + breviscapine (12 $\left.\mathrm{mg} \mathrm{kg}^{-1} ; p=0.039\right)$ groups (Table 6, Fig. 3a).

Table 4 Comparison of survival curves after lethal dose 13 Gy TBI $(n=10)$

\begin{tabular}{|c|c|c|c|c|}
\hline & \multicolumn{3}{|c|}{$\begin{array}{l}\text { Log-rank (Mantel-Cox) } \\
\text { test }\end{array}$} & \multirow{2}{*}{$\begin{array}{l}\text { sig difference? } \\
(p<0.05 \text { as } \\
\text { significance })\end{array}$} \\
\hline & Chi square & df & $p$ value & \\
\hline Control vs. bupleurum & 11.410 & 1 & 0.0007 & Yes \\
\hline Control vs. shenmai & 4.520 & 1 & 0.034 & Yes \\
\hline Control vs. herba houttuyniae & 3.473 & 1 & 0.062 & No \\
\hline Control vs. breviscapine & 4.620 & 1 & 0.032 & Yes \\
\hline $\begin{array}{l}\text { Control vs. sulfotanshinone } \\
\text { sodium }\end{array}$ & 0.3702 & 1 & 0.543 & No \\
\hline
\end{tabular}

Table 5 Comparison of 30 day survival curves after 7 Gy TBI $(n=10)$

\begin{tabular}{|c|c|c|c|c|}
\hline & \multicolumn{3}{|c|}{$\begin{array}{l}\text { Log-rank (Mantel-Cox) } \\
\text { test }\end{array}$} & \multirow{2}{*}{$\begin{array}{l}\text { Sig difference? } \\
(\mathrm{p}<0.05 \text { as } \\
\text { significance })\end{array}$} \\
\hline & Chi square & df & $\begin{array}{l}p \\
\text { value }\end{array}$ & \\
\hline Control vs. bupleurum & 4.405 & 1 & 0.036 & Yes \\
\hline Control vs. shenmai & 5.086 & 1 & 0.024 & Yes \\
\hline $\begin{array}{l}\text { Control } v s . \text { herba } \\
\text { houttuyniae }\end{array}$ & 3.440 & 1 & 0.064 & No \\
\hline Control vs. breviscapine & 3.913 & 1 & 0.048 & Yes \\
\hline Control vs. sulfotanshinone & 0.353 & 1 & 0.55 & No \\
\hline
\end{tabular}

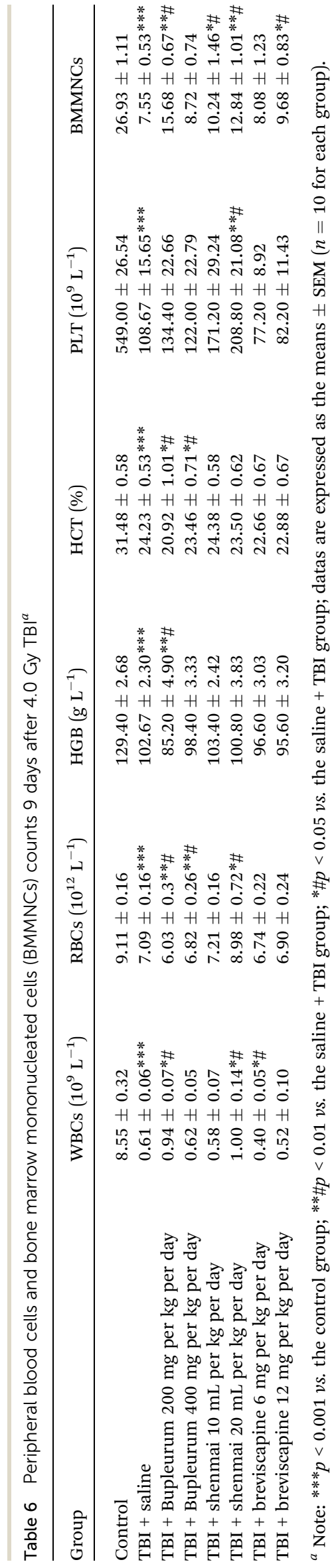


a

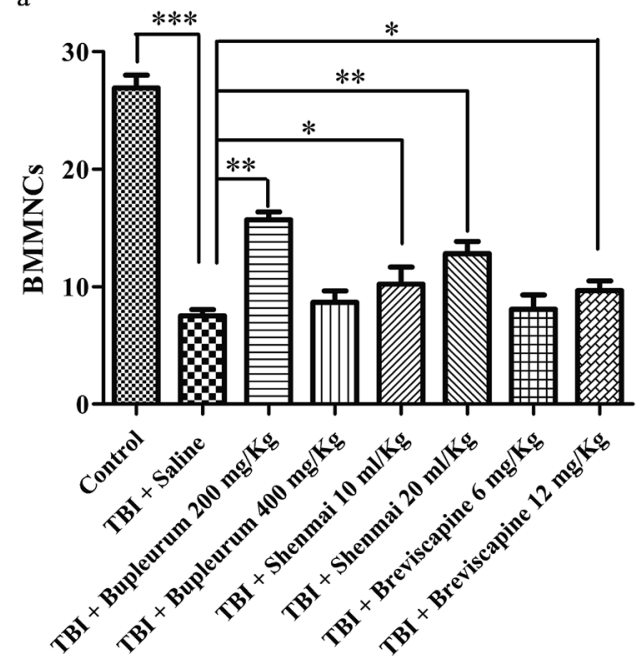

b

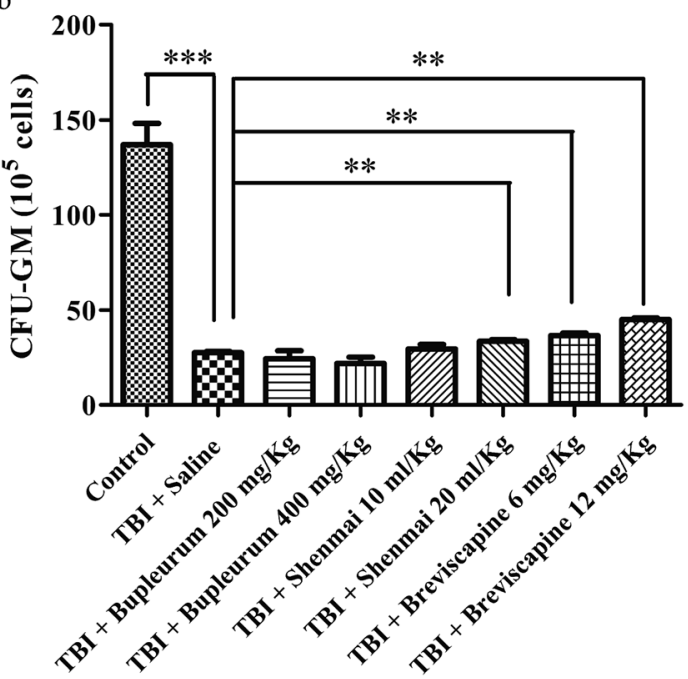

Fig. 3 Bupleurum injection, shenmai injection, and breviscapine injection attenuate low dose TBI-induced BM suppression. (a) 1 (bupleurum injection, $p=0.0012,0.27$ ), 3 (shenmai injection, $p=0.049,0.0038$ ), 7 (breviscapine injection, $p=0.96,0.039$ ) ameliorates sublethal dose TBIinduced BMMNCs decrease. (b) 3 (shenmai injection, $p=0.89,0.0026$ ), 4 (herba houttuyniae injection, $p=0.0018,0.0018$ ) attenuated TBIinduced CFU-GM reduction.

Irradiation not only induces a reduction in hematopoietic cell number, but also causes functional inhibition of cells. ${ }^{7-9}$ As a measure of hematopoietic function, we therefore evaluated the effects of TCMC on CFU-GM. In comparison with the TBI + saline group, the TBI + shenmai $\left(20 \mathrm{~mL} \mathrm{~kg}^{-1} ; p=0.0026\right)$ and TBI + breviscapine $\left(6 \mathrm{mg} \mathrm{kg}^{-1}\right.$ and $12 \mathrm{mg} \mathrm{kg}^{-1} ; p=0.0018, p=$ 0.0018 , respectively) groups showed a significant increase in CFU-GMs (Fig. 3b).

These results suggest that the hematopoietic cell functions affected by IR can be partially restored by treatment with bupleurum, shenmai, or breviscapine.

\section{Discussion}

TCM has been used in China for more than two thousand years in the clinical treatment of a wide range of diseases including cerebrovascular diseases, stroke, amnesia, and dementia. In recent years China, several new compounds have been developed based on classical TCM formulae, ${ }^{3,10}$ for the treatment of, for example, stroke and inflammation. ${ }^{11,12}$ In addition, Chinese materia medica as well as active ingredients and components included in TCMCs, are being increasingly investigated, ${ }^{13-15}$ including for radioprotective effects. ${ }^{16}$ In the present study, the radioprotective activity of 13 selected TCMCs were evaluated, the TCMCs had been used in clinical practice for the treatment of influenza and malaria (bupleurum), heart disease (Danshen, Shenmai, Xiangdan, Salvianolate, Sulfotanshinone sodium), tumors (shenmai, synergistically with chemotherapy), stroke (breviscapine), and cerebrovascular disease (safflower), and as anti-oxidants and anti-inflammatory agents (Radix isatidis, Chuanxinlian, Shuanghuanglian, Antihepatitis-jaundice compound). Clinical trials of the TCMCs have shown that they are active pharmacologically as anti-oxidants, antiinflammatory agents, and anti-microbials, and that they can act in isolation as well as in combination with other constituents. ${ }^{17-21}$ Our study, to the best of our knowledge, is the first to examine the therapeutic effects of these TCMCs on ionizing radiation damage in animal models.

\subsection{Five TCMCs demonstrated radioprotective effect with no cytotoxicity}

We first evaluated the cytotoxicity of TCMCs on 293T cells (Table 1) to determine the IC10 values and chose half IC10 as the appropriate concentration to investigate the cell response to different doses of IR exposure (Fig. 1). bupleurum injection, shenmai injection, herba houttuyniae injection, breviscapine injection, sulfotanshinone sodium injection demonstrated strong radioprotective effect without cytotoxicity. These five TCMCs have been used widely in clinical practice, bupleurum exhibits evident anti-inflammatory, antitumor, antiviral, anti-allergic, immunoregulation, and neuroregulation activities. ${ }^{22}$ Shenmai injection has been widely applied as an organ protector for cardio/ cerebrovascular diseases, viral myocarditis, tumor chemotherapy, and adverse drug reactions. ${ }^{23}$ Although with some known adverse drug events and adverse drug reactions, ${ }^{24}$ herba houttuyniae has been proven to have heat-clearing, detoxifying and neuroprotective effects. ${ }^{25}$ Breviscapine injection has been extensively used in China since the 1970s for the treatment of ischemic cardiovascular and cerebrovascular diseases, such as angina pectoris, myocardial infarction and focal cerebral infarction..$^{26}$ Sulfotanshinone sodium injection has been widely applied for its protective effect against angina pectoris $^{27}$ and atherosclerotic lesions. ${ }^{28}$

\subsection{TBI animal model}

TBI animal model was established to further assess the radioprotective effect of the five TCMCs to assess radioprotective 
effect of these five TCMCs, TBI animal model was established, as described by Rosen et al., ${ }^{29}$ TBI affects multiple organ systems or causes death, mainly via two mechanisms, gastrointestinal (GI) syndrome and hematopoietic syndrome. An effective radioprotector/mitigator should improve post-IR 30 day survival rates in rodents or humans by protecting against GI syndrome, hematopoietic syndrome, or both. The results from the present study showed that bupleurum, shenmai, and breviscapine effectively prolonged survival time of mice exposed to a supra-lethal (13 Gy) TBI dose, and improved the 30 day survival of mice exposed to a lethal (7 Gy) dose. The administration of each also attenuated sub-lethal TBI (4 Gy)-induced BM suppression, which was consistent with the results of the 293T cell colony formation assay. Evidence of hematopoietic protection was revealed by analysis of peripheral blood parameters after sub-lethal TBI. A significant increase in WBCs, RBCs, HGB and HCT was seen in bupleurum (200 mg kg ${ }^{-1}$ )-treated mice compared with those treated with saline, and treatment with shenmai $\left(20 \mathrm{~mL} \mathrm{~kg}^{-1}\right)$ similarly affected WBCs, RBCs and HCT. While, compared to treatment with saline, shenmai and breviscapine both had significant positive effects on CFU-GM formation after sub-lethal IR.

\subsection{Bupleurum, shenmai and breviscapine could be potentially used as potential radioprotectants}

These data indicate that bupleurum, shenmai and breviscapine can act as effective radioprotectants, but the underlying mechanisms of their possible roles in the pathogenesis of TBIinduced injury and the active components involved remain to be determined. Furthermore, in the context of cancer treatment, other specific aspects of TCMC use will require evaluation, including the fact that protectants can often selectively target normal rather than tumor tissue, ${ }^{29-31}$ and the possible synergistic effects with tumor radiotherapy. Elucidating the mechanisms of action of TCMCs remains a great challenge due to the lack of knowledge of the active components and the possibility of synergistic actions of multiple components with one compound. In the present study, we found that some TCMCs were apparently cytotoxic or even had an IR sensitization effect, however, this requires confirmation.

Another unexplored area is the longer-term whole body effects of these radioprotector candidates on, for example, the skin, esophagus, lung and kidney, which are more radiosensitive tissues than $\mathrm{BM}$ and intestine. An additional consideration is how long after exposure to radiation would the TCMCs be effective as there is usually a "window" of time to administer protection prior to or after radiation; ${ }^{29}$ obviously, an agent with a wide window would be most valuable.

\section{Conclusion}

Bupleurum injection, shenmai injection, and breviscapine injection can be used as an efficacious radiation damage rescuer, further studies are necessary to confirm the bioactive compounds responsible for the radioprotective effect.

\section{Conflict of interest}

The authors declare that there is no conflict of interests regarding the publication of this paper.

\section{List of abbreviations}

$\begin{array}{ll}\text { BMMNCs } & \text { Bone marrow mononucleated cells } \\ \text { CAMS } & \text { Chinese Academy of Medical Sciences } \\ \text { CFU-GM } & \text { Colony-forming units-granulocyte/macrophage } \\ \text { HCT } & \text { Red blood cell-specific volume } \\ \text { HGB } & \text { Hemoglobin } \\ \text { IC10 } & \text { 10 percent inhibitory concentration } \\ \text { IR } & \text { Irradiation } \\ \text { LD } & \text { Median lethal dose } \\ \text { MTT } & 3-(4,5-D i m e t h y l-2-\text { thiazolyl)-2,5-diphenyl-2- } H \text { - } \\ & \text { tetrazolium bromide } \\ \text { PE } & \text { Plating efficiency } \\ \text { PLT } & \text { Platelets } \\ \text { RBCs } & \text { Red blood cells } \\ \text { SF } & \text { Survival fraction } \\ \text { SI } & \text { Sham-irradiated } \\ \text { TBI } & \text { Total-body irradiation } \\ \text { TCM } & \text { Traditional Chinese medicine } \\ \text { TCMC } & \text { Traditional Chinese medicine compound } \\ \text { WBCs } & \text { White blood cells. }\end{array}$

\section{Acknowledgements}

This paper was supported by National Natural Science Foundation of China (No. 81402541, No. 81403059), the PUMC Youth Fund (3332016144) and the Fundamental Research Funds for CAMS (No. 1608).

\section{References}

1 M. Z. Kamran, A. Ranjan, N. Kaur, S. Sur and V. Tandon, Med. Res. Rev., 2016, 36, 461-493.

2 C. N. Coleman, B. W. Fau, J. R. Fike, T. J. MacVittie and R. S. Wong, Radiat. Res., 2003, 159, 812-834.

3 K. Sun, J. Fan and J. Han, Acta Pharm. Sin. B, 2015, 5, 8-24. 4 K. J. Krager, E. N. Pineda, S. V. Kharade, M. Kordsmeier, L. Howard, P. J. Breen, C. M. Compadre, M. Hauer-Jensen and N. Aykin-Burns, Evid. base. Compl. Alternative Med., 2015, 2015, 148791.

5 S. Reagan-Shaw, M. Nihal and N. Ahmad, FASEB J., 2008, 22, 659-661.

6 D. J. Brenner, Semin Radiat Oncol, 2008, 18, 234-239.

7 A. Meng, Y. Wang, G. Van Zant and D. Zhou, Cancer Res., 2003, 63, 5414-5419.

8 Y. Wang, B. A. Schulte, A. C. LaRue, M. Ogawa and D. Zhou, Blood, 2006, 107, 358-366.

9 P. Mauch, L. Constine, J. Greenberger, W. Knospe, J. Sullivan, J. L. Liesveld and H. J. Deeg, Int. J. Radiat. Oncol., Biol., Phys., 1995, 31, 1319-1339. 
10 X. Meng, M. Wang, X. Wang, G. Sun, J. Ye, H. Xu and X. Sun, Free Radical Res., 2014, 48, 823-838.

11 J. Li, J. Xu, W. Xu, Y. Qi, Y. Lu, L. Qiu, Z. Hu, Z. Chu, Y. Chai and J. Zhang, Int. J. Mol. Sci., 2015, 16, 18938-18955.

12 D. Li, L. Lu, J. Zhang, X. Wang, Y. Xing, H. Wu, X. Yang, Z. Shi, M. Zhao, S. Fan and A. Meng, Int. J. Mol. Sci., 2014, 15, 10541-10553.

13 V. Veerapur, K. Prabhakar, V. Parihar, M. Kandadi, S. Ramakrishana, B. Mishra, B. Satish Rao, K. Srinivasan, K. Priyadarsini and M. Unnikrishnan, Evid. base. Compl. Alternative Med., 2009, 6, 317-324.

14 A. Montoro, J. F. Barquinero, M. Almonacid, A. Montoro, N. Sebastia, G. Verdu, V. Sahuquillo, J. Serrano, M. Saiz, J. I. Villaescusa and J. M. Soriano, Evid. base. Compl. Alternative Med., 2011, 2011, 174853.

15 M. Bala, M. Gupta, M. Saini, M. Z. Abdin and J. Prasad, Evid. base. Compl. Alternative Med., 2015, 2015, 765705.

16 L. He, J. Wang, B. Sun, J. Zhao, L. Li, T. Xu, H. Li, J. Sun, J. Ren, R. Liu, Q. Chen, Z. Zhang and Y. Li, J. Nutr. Biochem., 2017, 40, 53-61.

17 X. H. Zhu, S. J. Li, H. H. Hu, L. R. Sun, M. Das and T. M. Gao, J. Ethnopharmacol., 2010, 127, 38-46.

18 L. Li, J. S. Wang and L. Kong, Chin. J. Nat. Med., 2013, 11, 222-230.

19 X. S. Xu, Z. Z. Ma, F. Wang, B. H. Hu, C. S. Wang, Y. Y. Liu, X. R. Zhao, L. H. An, X. Chang, F. L. Liao, J. Y. Fan, H. Niimi and J. Y. Han, Shock, 2009, 32, 201-209.
20 W. Ding, J. Gu, L. Cao, N. Li, G. Ding, Z. Wang, L. Chen, X. Xu and W. Xiao, J. Ethnopharmacol., 2014, 155, 589-598.

21 P. Lam, F. Cheung, H. Y. Tan, N. Wang, M. F. Yuen and Y. Feng, Int. J. Mol. Sci., 2016, 17, 465.

22 B. Yuan, R. Yang, Y. Ma, S. Zhou, X. Zhang and Y. Liu, Pharm. Biol., 2017, 55, 620-635.

23 L. Y. Lu, G. A.-O. Zheng and Y. Wang, Evid base Compl Alternative Med, 2014, 2014, 840650.

24 Z. X. Bian, L. Gao, H. C. Shang, T. X. Wu, Y. P. Li, B. L. Zhang and L. Song, J. Evid. Base. Med., 2010, 3, 5-10.

25 H. G. Kim, H. U. Jeong, S. I. Hong and M. S. Oh, Planta Med., 2015, 81, 1697-1704.

26 X. Liu, L. Yao, D. Sun, X. Zhu, Q. Liu, T. Xu and L. Wang, Exp. Ther. Med., 2016, 12, 1383-1397.

27 X. Qiu, X. Jiang, X. Sun and N. Yang, Evid. base. Compl. Alternative Med., 2012, 2012, 715790.

28 W. Zhang, J. Liu, J. Wang, S. Zhang, S. Zhang and Z. Wu, Biomaterials, 2013, 34, 306-319.

29 E. M. Rosen, R. Day and V. K. Singh, Front. Oncol., 2014, 4, 381.

30 S. Fan, Q. Meng, J. Xu, Y. Jiao, L. Zhao, X. Zhang, F. H. Sarkar, M. L. Brown, A. Dritschilo and E. M. Rosen, Proc. Natl. Acad. Sci. U. S. A., 2013, 110, 18650-18655.

31 R. M. Day, T. A. Davis, M. Barshishat-Kupper, E. A. McCart, A. J. Tipton and M. R. Landauer, Int. Immunopharmacol., 2013, 15, 348-356. 